\title{
Interaction of recombinant leukocyte A interferon with lipid monolayers
}

\author{
N.A. Williams * and N.D. Weiner \\ College of Pharmacy, University of Michigan, Ann Arbor, MI 48109 (U.S.A.)
}

(Received 15 August 1988)

(Modified version received 13 October 1988)

(Accepted 17 October 1988)

Key words: Lipid monolayer; Leukocyte A interferon

\section{Summary}

The extent of interaction of recombinant leukocyte A interferon (IFN- $\alpha$ ) with neutral, positively charged and negatively charged phospholipid monomolecular films was studied by measuring its ability, after injection into the subphase, to increase surface pressures exerted by such lipid films. The maximum surface pressure exerted by IFN- $\alpha$ in the absence of a lipid film (22.6 dyne/cm) was found to be nearly identical to the critical pressure for penetration of IFN- $\alpha$ into each of the lipid films studied. Thus, for lipid films at surface pressures above this value, there was no measurable penetration by IFN- $\alpha$. At lower surface pressure values, injection of IFN- $\alpha$ into the subphase resulted in an increase in pressure, indicating its penetration into the film. Since the surface pressure exerted by natural and artificial bilayer structures is greater than $22.6 \mathrm{dyne} / \mathrm{cm}$, extrapolation of these observations suggests that IFN- $\alpha$ would be excluded from the lipid bilayer of liposomes.

Interferon- $\alpha$ (IFN- $\alpha$ ) is an immunomodulator whose antiviral and antiproliferative effects are well-established (Baron et al., 1987). Successful entrapment of IFN- $\alpha$ in liposomes (Anderson et al., 1981; Eppstein and Stewart, 1981, 1982) has encouraged attempts to use the latter as a drug delivery system for the protein. For example, it has been shown that when IFN- $\alpha$ is encapsulated in liposomes and injected intramuscularly in mice, interferon is retained at the site of injection for much longer times than free IFN- $\alpha$ is (Eppstein and Stewart, 1982). The dependence of the ability

\footnotetext{
* Present address. Liquid Products Department, Abbott Laboratories, North Chicago, IL 60064, U.S.A.

Correspondence: N.D. Weiner, College of Pharmacy, University of Michigan, Ann Arbor, MI 48109, U.S.A.
}

of liposomes to entrap interferon, on the lipid composition, prompts the need to study the protein's interactions with lipid assemblies. One method which has been used to study polypeptide-lipid interactions is the monolayer technique in which a lipid is spread on the surface of a buffer, the polypeptide is injected into the subphase, and the extent of the resulting interaction is determined by measuring the change in surface pressure (Kimelberg and Papahadjopoulos, 1971; Schwinke et al., 1983). Such techniques provide insight into protein interactions with artificial (Kimelberg and Papahadjopoulos, 1971) and natural (Bougis et al., 1981) lipid bilayers. For example, protein-lipid bilayer interactions will affect the location of the encapsulated protein in lipid vesicles, which may in turn affect the ability of the 
vesicles to retain the entrapped protein over a period of time. An understanding of such interactions therefore may be valuable in selecting a suitable lipid composition as a delivery system for the protein.

In this study, we employ the constant-area monolayer technique to investigate the interactions between recombinant leukocyte $\mathrm{A}$ interferon (IFN- $\alpha$ ) with some selected lipid monolaycrs. Dimyristoylphosphatidylcholine (DMPC), stearylamine (SA) and phosphatidylserine (PS; Avanti Polar Lipids, Pelham, AL) were used as received. The purity of each of these lipids was $>99 \%$ as determined by thin-layer chromatography. Recombinant leukocyte A interferon (IFN- $\alpha$ ), supplied by Hoffmann-La Roche (Nutley, NJ) consists of 165 amino acids with a calculated molecular molecular weight of 19,219 (Pestka, 1983).

The monolayer penetration studies were performed by a modification of a procedure reported by Schwinke et al. (1983). The following lipid mixtures were used (mole ratios are given in parentheses): DMPC: PC $(2: 1)$ and DMPC:SA $(2: 1)$. The experiments were performed at $25 \pm$ $1^{\circ} \mathrm{C}, \approx 2^{\circ} \mathrm{C}$ above the phase transition of DMPC (Janiak et al., 1976). A Rosano Surface Tensiometer (Laboratory Products, Boston, MA) having a sensitivity of 0.05 dyne $/ \mathrm{cm}$, equipped with a sandblasted platinum Wilhelmy plate was used to measure surface tension. The subphase consisted of $90.0 \mathrm{ml}$ of $0.05 \mathrm{M} \mathrm{N}$-2-hydroxyethylpiperazine$N^{\prime}$-2-cthancsulphonic acid (HEPES) at $\mathrm{pH} 7.0$. Sodium chloride was added to adjust the ionic strength to 0.2 . The solution was contained in a circular polytetrafluoroethylene dish $7.63 \mathrm{~cm}$ in internal diameter. A polytetrafluoroethylenecoated magnetic stirrer was used for mixing the subphase $(\approx 80 \mathrm{rpm})$ without disturbing the surface. The entire apparatus was enclosed to reduce airborne contamination.

The pure lipid or lipid mixture was spread from a solution of $n$-hexane: ethanol $(9: 1, v / v)$ by means of an Agla micrometer syringe (Wellcome Reagents, Beckenham, U.K.) in amounts sufficient to produce the desired initial surface pressure. The addition of the spreading solvent alone to a clean buffer surface produced no measurable change in surface tension.
A stationary needle with a removable glass syringe was used to deliver $460 \mu \mathrm{g}$ IFN $-\alpha$ in 0.2 $\mathrm{ml}$ of solution beneath the surface into the subphase so that the final concentration of IFN- $\alpha$ was $5.1 \mu \mathrm{g} / \mathrm{ml}$. An equal volume of the subphase was removed prior to the addition so as to keep the total volume constant. Surface tension readings were taken every $5 \mathrm{~min}$ until no further changes could be observed $(<0.05 \mathrm{dyne} / \mathrm{cm}$ ) for $30 \mathrm{~min}$. In almost all cases, surface tension changes due to protein addition were virtually complete within $30 \mathrm{~min}$.

Surface pressure, $\pi$, was calculated as the difference in surface tension in the absence of the lipid film and that of the film-covered surface. The change in surface pressure, $\Delta \pi$, was calculated as the difference in surface pressure of the lipid film upon injection of protein into the subphase and that of the film in the absence of protein, i.e. at its initial surface pressure.

When increasing amounts of IFN- $\alpha$ were introduced into the subphase in the absence of a lipid monolayer, the measured surface pressures approached an asymptotic value at high IFN- $\alpha$ concentrations. Extrapolation to infinite IFN- $\alpha$ concentration of a plot of surface pressure vs reciprocal concentration gave a limiting pressure of 22.6 dyne/cm (Fig. 1). For the lipid monolayer penetration experiments, a working IFN- $\alpha$ con-

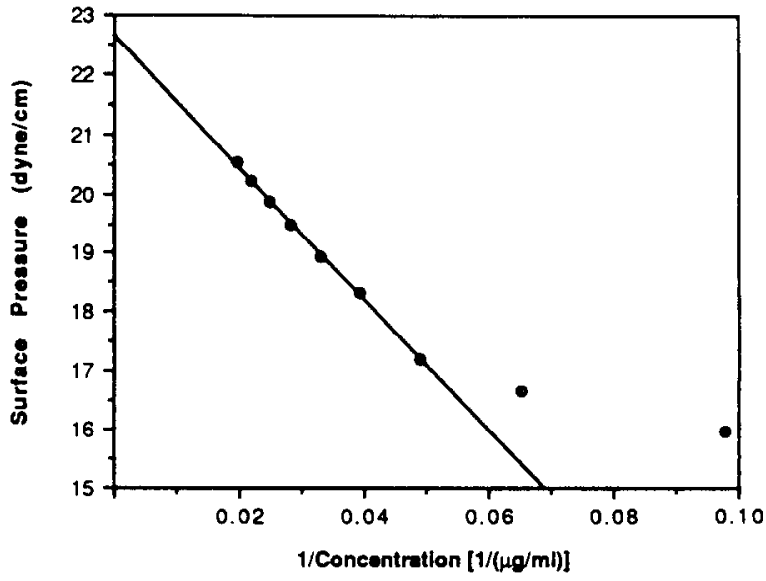

Fig. 1. Determination of the limiting pressure of IFN- $\alpha$ in the absence of a lipid film by extrapolation to infinite concentration of a plot of surface pressure as a function of IFN- $\alpha$ reciprocal concentration. 


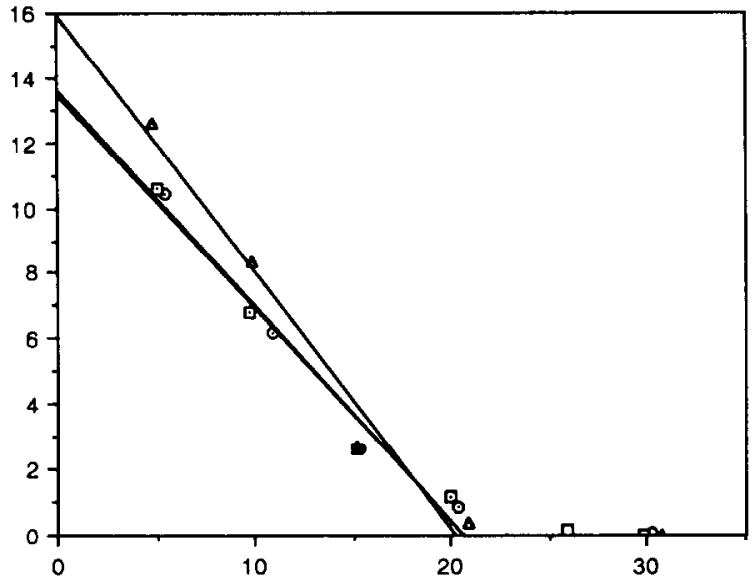

Fig. 2. Plots of the change in surface pressure as a function of initial surface pressure for various lipid monolayers upon injection of sufficient IFN- $\alpha$ to produce a final protein concentration of $5.1 \mu \mathrm{g} / \mathrm{ml}$. See text for experimental details. $\square$, DMPC; $\circlearrowleft$, DMPC : SA $(2: 1) ; \triangle$, DMPC: PS $(2: 1)$.

centration of $5.1 \mu \mathrm{g} / \mathrm{ml}$ was chosen because this concentration yielded a surface pressure of about 15 dyne $/ \mathrm{cm}$, equivalent to about $70 \%$ of the limiting pressure.

Fig. 2 shows the change in surface pressure caused by IFN- $\alpha$ as a function of initial surface pressure for 3 different lipid monolayers. In each case, as the initial surface pressure increases, $\Delta \pi$ decreases until it becomes zero at approximately $20 \mathrm{dyne} / \mathrm{cm}$. This value is slightly less than the limiting pressure of the protein film. As seen in Fig. 2, this trend appears, under the conditions of the experiment, to be independent of lipid charge suggesting that IFN- $\alpha$ is unable to penetrate the lipid monolayers used when these are spread at pressures greater than its own limiting pressure.

An implication of these observations is that, although IFN- $\alpha$ is quite surface-active by itself (mellitin, considered one of the most surface-active biological compounds (Bougis et al., 1981), has a limiting pressure of $24.5 \mathrm{dyne} / \mathrm{cm}$ (Sessa et al., 1969)), it seems to lack the features necessary for penetration into lipid films at surface pressures above its own limiting pressure. It has been known for some time that proteins and polypeptides which can penetrate lipid monolayers well above their limiting pressures, i.e. at pressures of about 30 dyne $/ \mathrm{cm}$ or more, can cause cell lysis (Bougis et al., 1981) increased permeability to ions (Kimelberg and Papahadjopoulos, 1971; Sessa et al., 1969) and may be found associated with biological membranes (Shiffer et al., 1988). Conversely, those proteins which are unable to penetrate lipid monolayers above their limiting pressures do not exhibit any of these properties. IFN- $\alpha$ appears to be one such protein. It should be pointed out though, that the ability of a protein to interact with a monolayer is dependent on a number of parameters, the most important being the charge on the lipid and the subphase $\mathrm{pH}$ and ionic strength (Kimelberg and Papahadjopoulos, 1971; Khaiat and Miller, 1969). Therefore, extrapolations of monolayer results to biological effects should be made with caution.

It has been proposed and demonstrated in several cases (Segrest and Feldmann, 1974) that proteins possessing continuous sequences of at least 10 uncharged amino acid residues with a hydrophobic index of about 2.7 and above, are likely to possess membrane-penetrating abilities. For IFN- $\alpha$, the hydrophobic sequences with the largest hydrophobic indices are 14-21, 24-30, $59-70$ and $97-106$ with values of $1.88,2.29,2.05$ and 1.45 , respectively. These values fall well within the range where membrane penetration is not expected and this is borne out by the results obtained in this study.

MacDonald and Simon (1987) have provided evidence that the molecular packing and lateral pressures of lipid bilayers and monolayers are closely related. In addition, they have shown that the surface tension of a DMPC monolayer in equilibrium with bilayers of multilamellar vesicles is 23 dyne $/ \mathrm{cm}$, corresponding to a surface pressure of $49 \mathrm{dyne} / \mathrm{cm}$. This pressure is at least twice the pressure at which the DMPC monolayer "squeezes out" IFN- $\alpha$. Thus, there is good reason to expect that IFN- $\alpha$, when entrapped in DMPC liposomes (and probably in other lipid vesicles) will be confined to the aqueous compartments between the lipid bilayers. This, of course, does not preclude interferon's association with the lipid bilayer, but simply means that the polypeptide is not in an area-determining position. It has been previously reported (Eppstein and Stewart, 1981, 1982) that human IFN- $\alpha$ is entrapped in lipo- 
somes but it was unclear whether the polypeptide was in the aqueous compartments only or intercalated within the lipid bilayers as well. The evidence presented here suggests strongly that very little, if any, interferon would penetrate into the bilayer.

\section{Acknowledgements}

This study was supported by Grant AI/ GM22303 from the National Institutes of Health.

\section{References}

Anderson, P., Vilcek, J. and Weissmann, G., Entrapment of human leukocyte interferon in the aqueous interstices of liposomes. Infect. Immun., 31 (1981) 1099-1103.

Baron, S., Stanton, G.J., Fleischmann, Jr., W.R. and Dianzani, $F$, Introduction: general considerations of the interferon system. In Brown, S., Dianzani, F., Stanton, G.J. and Fleischmann Jr., W.R. (Eds.), The Interferon System: a Current Review to 1987, University of Texas Press, Austin, TX, 1987.

Bougis, P., Rochat, H., Pieroni, G. and Verger, R., Penetration of phospholipid monolayers by cardiotoxins. Biochemistry, 20 (1981) 4915-4920.

Eppstein, D.A. and Stewart II, W.E., Binding and capture of human interferon- $\alpha$ by reverse evaporation vesicles, multilamellar vesicles, and small unilamellar vesicles. J. Interferon Res., 1 (1981) 495-505.

Eppstein, D.A. and Stewart II, W.E., Altered pharmacological properties of liposome-associated human interferon- $\alpha . J$. Virol., 41 (1982) 575-582.

Janiak, M.J., Small, D.M. and Shipley, G.G., Nature of the thermal pretransition of synthetic phospholipids: dimyrisistoyl- and dipalmitoyl-lecithin. Biochemistry, 15 (1976) 4575-4580.

Khaiat, A. and Miller, R., Adsorption of ribonuclease at the air-water interface and on phospholipid monolayers. Biochim. Biophys. Acta, 183 (1969) 309-319.

Kimelberg, H.K. and Papahadjopoulos, D., Phospholipid-protein interactions: membrane permeability correlated with monolayer penetration. Biochim. Biophys. Acta, 233 (1971) 805-809.

MacDonald, R.C. and Simon, S.A., Lipid monolayer states and their relationships to bilayers. Proc. Natl. Acad. Sci. U.S.A., 84 (1987) 4089-4093.

Pestka, S., The human interferons - from protein purification and sequence to cloning and expression in bacteria: before, between, and beyond. Arch. Biochem. Biophys, 221 (1983) 1-37.

Peterson, G.L., A simplification of the protein assay method of Lowry et al. which is more generally applicable. Anal. Biochem., 83 (1971) 346-356.

Schwinke, D., Ganesan, M.C. and Weiner, N.D., Monolayer studies of insulin-lipid interactions. J. Pharm. Sci., 72 (1983) 244-248.

Segrest, J.P. and Feldmann, R.J., Membrane proteins: amino acid sequence and membrane penetration. J. Mol. Biol., 87 (1974) 853-858.

Sessa, G., Freer, J.HI., Colacicco, G. and Weissman, G., Interaction of alytic polypeptide, melittin, with lipid membrane systems. J. Biol. Chem. 244 (1969) 3575-3582.

Shiffer, K.A., Goerke, J., Duzgunes, N., Fedor, J. and Shohet, S.B., Interaction of erythrocyte protein 4.1 with phospholipids. A monolayer and liposome study. Biochim. Biophys. Acta, 937 (1988) 269-280. 